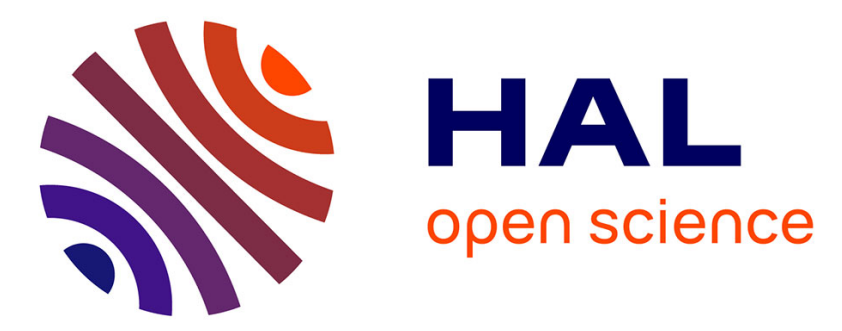

\title{
Uncertainties in the estimation of low frequency noise level extracted from noise spectral density measurements
}

Bruno Guillet, Sheng Wu, Bogdan Cretu, Rachida Talmat, H. Achour, Carlo Barone, S. Pagano, E. Sassier, Jean-Marc Routoure

\section{- To cite this version:}

Bruno Guillet, Sheng Wu, Bogdan Cretu, Rachida Talmat, H. Achour, et al.. Uncertainties in the estimation of low frequency noise level extracted from noise spectral density measurements. Noise and Fluctuations (ICNF), 2011 21st International Conference on,INCF, Jun 2011, Toronto, Canada. 4 p. hal-00993924

\section{HAL Id: hal-00993924 \\ https://hal.science/hal-00993924}

Submitted on 22 Jul 2014

HAL is a multi-disciplinary open access archive for the deposit and dissemination of scientific research documents, whether they are published or not. The documents may come from teaching and research institutions in France or abroad, or from public or private research centers.
L'archive ouverte pluridisciplinaire HAL, est destinée au dépôt et à la diffusion de documents scientifiques de niveau recherche, publiés ou non, émanant des établissements d'enseignement et de recherche français ou étrangers, des laboratoires publics ou privés. 


\title{
Uncertainties in the estimation of low frequency noise level extracted from noise spectral density measurements
}

\author{
Bruno Guillet*, Sheng Wu*, Bogdan Cretu*, Rachida Talmat* ${ }^{*}$, Hachim Achour* ${ }^{*}$, Carlo Barone ${ }^{\dagger}$, \\ Sergio Pagano ${ }^{\dagger}$, Eric Sassier* and Jean-Marc Routoure* \\ * GREYC, UMR CNRS 6072, ENSICAEN, Université de Caen Basse-Normandie, \\ 6 Boulevard du maréchal Juin, 14050 Caen Cedex, France \\ contact:jean-marc.routoure@unicaen.fr \\ † Dipartimento di Matematica e Informatica, CNR-SPIN Salerno, Università di Salerno, \\ 84084 Fisciano, Salerno, Italy \\ $\ddagger$ GRMNT, Mouloud Mammeri University of Tizi-Ouzou, Algeria
}

\begin{abstract}
It is well known that measurement uncertainty of noise spectral density is related to the time length of observation. With FFT spectrum analyzers, the measurement error can be reduced by averaging $N_{\text {avg }}$ measurements. Standard error is then reduced by the square root of $N_{a v g}$. This paper deals with the error introduced when one wants to extract from the noise spectral density measurement the $1 / \mathrm{f}$ level, white noise level and lorentzian parameters. In that purpose, different estimation techniques from different groups are compared and discussed.
\end{abstract}

\section{INTRODUCTION}

In this paper, several and different techniques used to estimate the extraction of $1 / \mathrm{f}$ noise level from a power spectrum analyzer are compared in terms of uncertainties. Each technique introduces different sources of error that can be quantified by the uncertainties $u(E i)$. The expanded uncertainty $u$ with a coverage factor $k$ is then given by $u=k \cdot \sqrt{\sum_{i} u(E i)^{2}}$ [1]. After a brief description of uncertainty estimation and the different ways used by the different groups to estimate the noise, the values of the uncertainty are given and discussed.

\section{UNCERTAINTY ESTIMATION}

Different groups involved in this work performed several times the same analysis on two kinds of voltage noise spectral density data $S_{V}(f)$ (figure $1 \mathrm{a}$ and b):

- spectrum A which mainly consists in white noise and $1 / \mathrm{f}$ noise with a corner frequency around $100 \mathrm{~Hz}$ (data obtained one oxide thin film),

- spectrum B with low frequency noise (1/f noise and several lorentzian spectra), white noise and a high corner frequency value close to $100 \mathrm{kHz}$ (data obtained from advanced MOS transistors).

For each spectrum, several parameters have been extracted: the white noise level $W$, the $1 /$ f noise level at $1 \mathrm{~Hz}$ $K$, the number of lorentzian $N_{L}$, the plateau $a_{i}$ and the a : spectrum A from oxide thin film

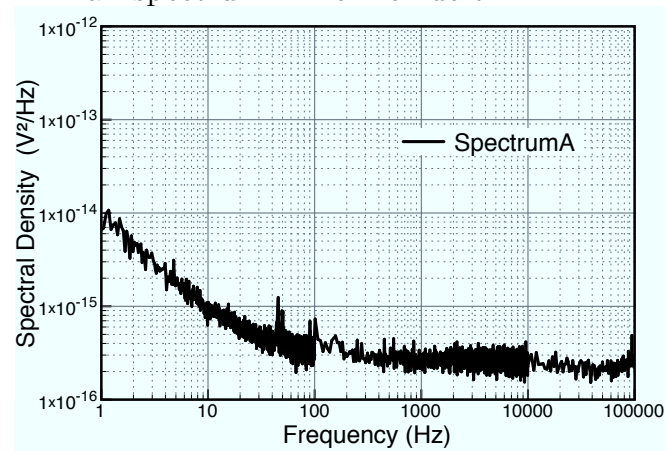

b. spectrum B from MOS transistor

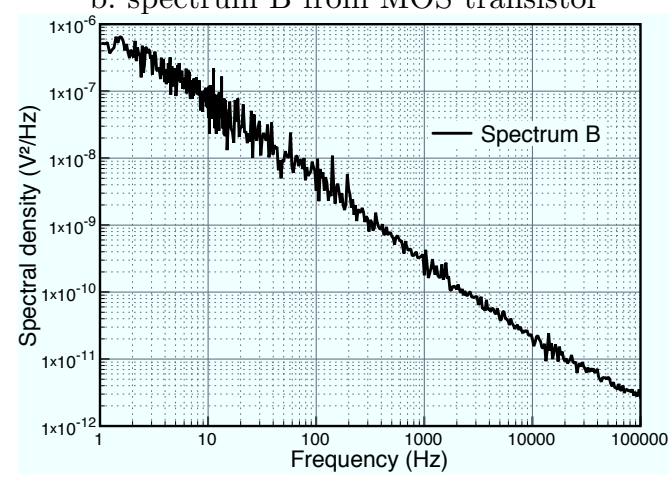

Fig. 1. Experimental voltage noise spectral densities used to investigate measurement uncertainty. The spectrum A (a) mainly consists in white noise with a low corner frequency whereas the spectrum B (b) exhibits mainly flicker noise with both $1 / \mathrm{f}$ noise and lorentzian noise.

characteristic frequency $f_{i}$ of each lorentzians according to the following equation:

$$
S_{V}(f)=W+\frac{K}{f}+\sum_{i=1}^{N_{L}} \frac{a_{i}}{1+\left(\frac{f}{f_{i}}\right)^{2}}
$$


This extraction has been performed $\mathrm{N}$ times (here 7 ). In order to minimize memory effects which could bias the results by introducing some correlation, these extractions have been done using a quite long period between 2 consecutive estimations and without reading the previous extracted values. The different techniques used by the different group are described in the next section.

Type A method (using statistical analysis of observation series) has then been used for the uncertainty estimation of the repetability error [1] for each method : mean value $(<$ $X>)$ and standard deviation $\left(\sigma_{X}\right)$ of each parameter has then been calculated according to the following standard definitions :

$$
<X>=\frac{\sum_{i=1}^{N} X_{i}}{N} \text { and } \sigma_{X}=\sqrt{\frac{\sum_{i=1}^{N}\left(X_{i}-<X>\right)^{2}}{N}}
$$

\section{Description of the Different estimation TECHNIQUES}

From all the techniques performed by the different research teams, one can distinguish:

- manual estimation (noted Hand1 and Hand2) using a log-log plot of each spectra on 7 different sheets of paper. A design rule was used to estimate the different parameters. Hand1 method used log-log plot of the spectral density $S_{V}(f)$ whereas Hand2 used log-log plot of the spectral density normalized by the frequency $S_{V}(f) \cdot f$,

- comparison of measurements and computed values according to equation (1). The choice of the best fits is related to a human operator and not to a computer elaboration. Several plotting or computing softwares (named Labview, Mathcad and Gnuplot) were used. In some softwares (Labview and Gnuplot), slides or cursors were available to improve the user interface. For Mathcad, the same algorithm was used by two different persons (named Mathcad1 and Mathcad2),

- use of a decomposition algorithm [2] : the spectra are approximated with a linear combination of sample functions : one or several $1 / f^{\gamma i}$ functions with $1 \leqslant \gamma i \leqslant 2$, and a family of Lorentzian functions with characteristic frequencies $\left(f_{i}\right)$ logarithmically distributed over the range of the studied spectrum. Once the sample functions are chosen, the linear combination of these functions which shows, on one hand, only positive coefficients and, on the other hand, the minimal distance to the approximated spectrum, is algebraically calculated. Since this approximation method is deterministic, repeating it several times cannot bring any further refinement. However, several calculation results are provided for each spectrum, obtained by varying in the sample functions family.

\section{Results And Discussion}

The results from the different techniques are plotted in the following figures for each parameters and each spectra.
The mean value of each parameters for all the techniques $\overline{\langle X\rangle}$ assuming the same weight as well as errors bars showing the repetability error given by $(2$ or 3$) \cdot \sigma_{X}$ of each techniques are also plotted. According to [1], the probability to find the value in the range indicated by the error bars is equal to $95 \%$ when coverage factor $\mathrm{k}=2$ and $98 \%$ when coverage factor $k=3$. It will then be assumed in the following discussion that the mean value gives a correct estimation of the real value of the concerned parameter. For one technique, when error bars do not cross this mean value line, it may be interpreted as a bias introduced by the technique. The case of the decomposition algorithm will be presented and discussed in a separated way.
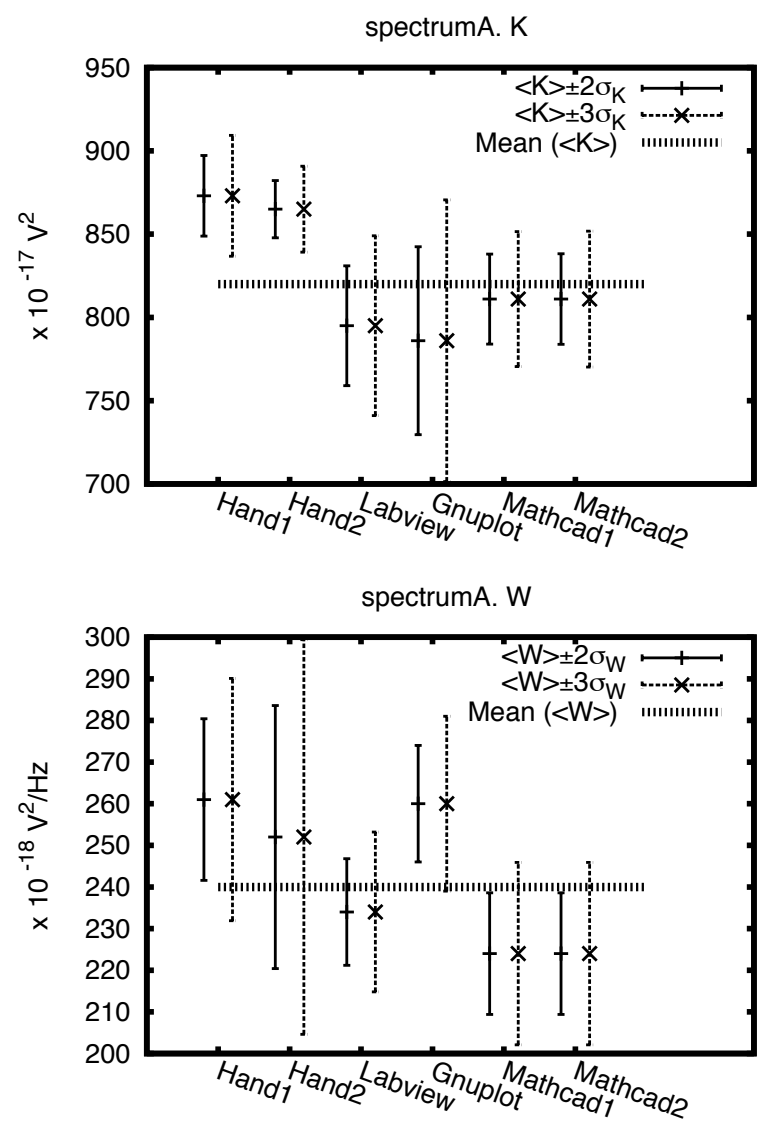

Fig. 2. SpectrumA results for parameters $\mathrm{K}$ and W. $\langle X\rangle \pm 2 \cdot \sigma_{X}$, $\langle X\rangle \pm 3 \cdot \sigma_{X}$ and $\overline{\langle X\rangle}$ for all the techniques.

From all these results, several remarks and comments can be proposed : $K$ and $W$ parameters are often overestimated by manual techniques.

No lorentzians were observed for the spectrumA for all the technique. Up to three lorentzians were identified using the "software" techniques for the spectrumB. Only the results for the first two lorentzians are reported here. Only one lorentzian was observed for the manual techniques 

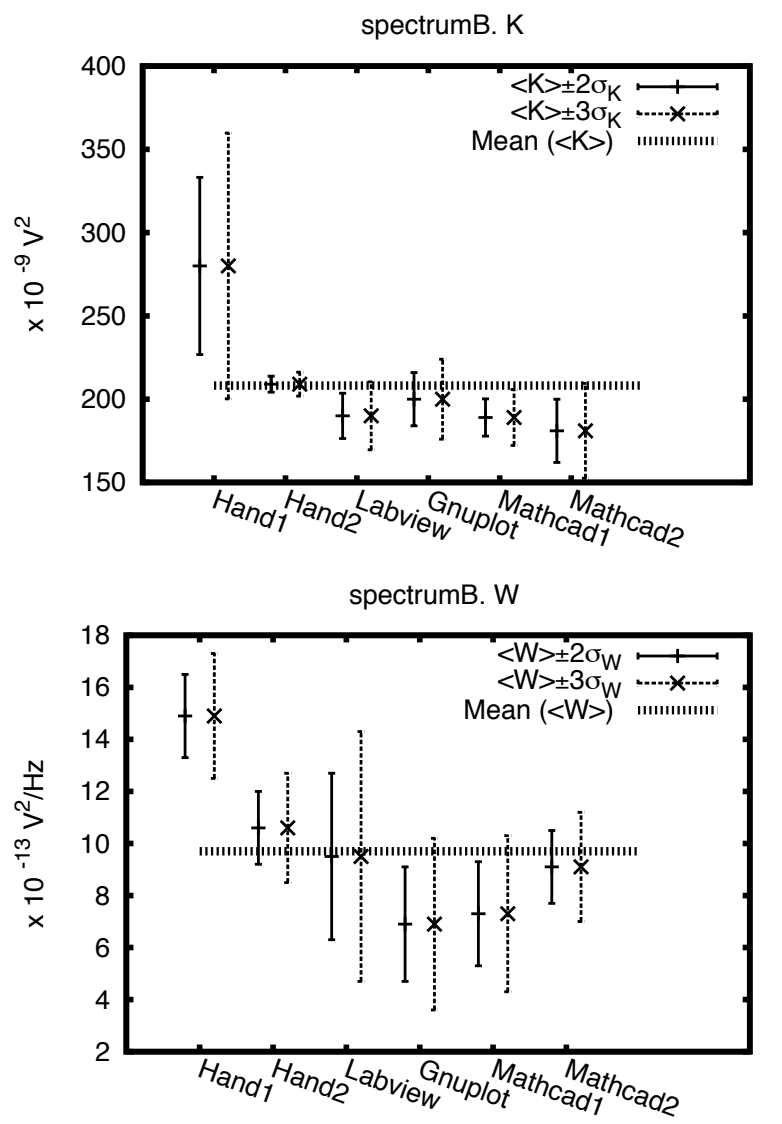

Fig. 3. SpectrumB results for parameters $\mathrm{K}$ and W. $\langle X\rangle \pm 2 \cdot \sigma_{X}$, $\langle X\rangle \pm 3 \cdot \sigma_{X}$ and $\overline{\langle X\rangle}$ for all the techniques.

with a large value of uncertainty. The Lorentzians appear as bumps in the spectral density difficult to quantify or estimate with a right design rule.

Except manual estimations, all the parameter estimations performed by software techniques give almost the same result with the almost the same repetability. This remark is not true for the second lorentzian of the spectrum B mainly due to its smallest weight in the total spectrum. The case of small repeatability uncertainty values for the manual technique in the case of the $\mathrm{K}$ parameter in spectrumB may be due to the fact that the value of $K$ is close to a value close to the tick mark of the graph.

A case of special interest is the errors in the estimation of the characteristic frequencies of lorentzians because when noise measurements are performed versus temperature, it allows to identify traps by using a method called "noise spectroscopy" (see [3] in this proceeding for instance). Whatever the software method used, $3 \cdot \sigma_{f_{1}} /\left\langle f_{1}\right\rangle$ is less than $27 \%$ and $3 \cdot \sigma_{f_{2}} /<f_{2}>$ is less then $42 \%$. Even if these values seems relatively high, when the noise spectroscopy method is applied, the characteristic frequency may vary over five decades of frequency ! fortunately, these relatively high uncertainty values may not thus affect the identification of the traps.
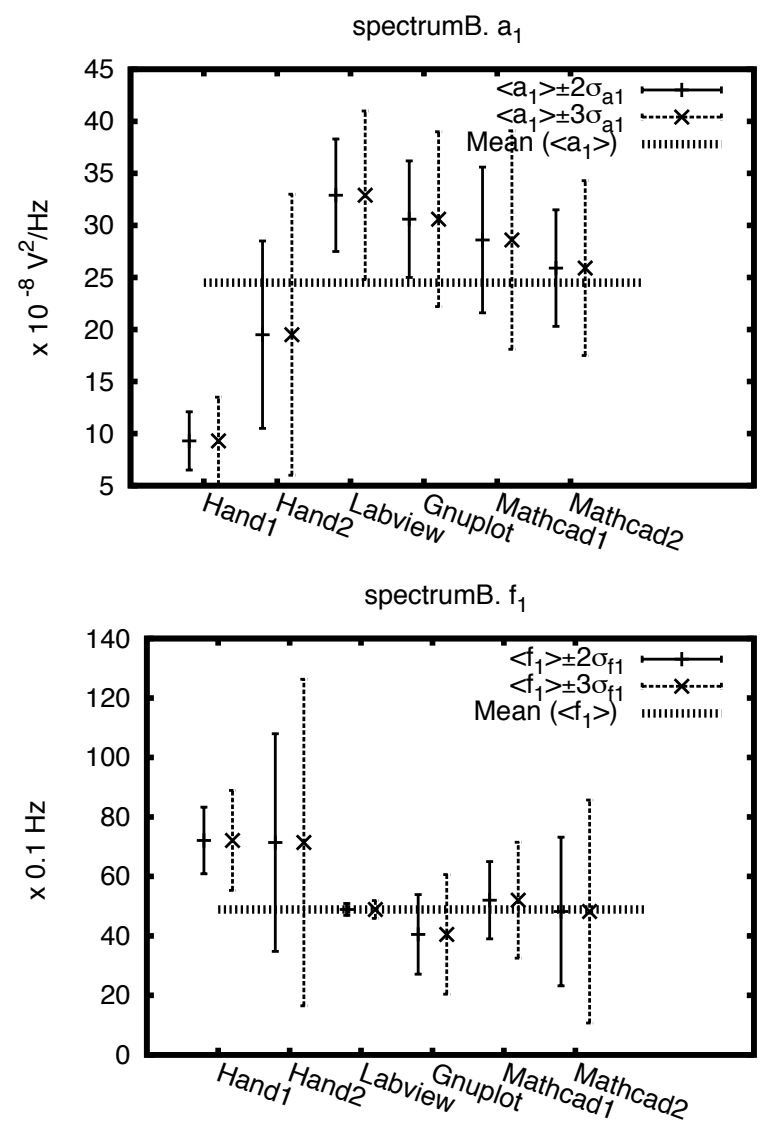

Fig. 4. SpectrumB results for parameters $a_{1}$ and $f_{1} .\langle X\rangle \pm 2 \cdot \sigma_{X}$, $\langle X\rangle \pm 3 \cdot \sigma_{X}$ and $\overline{\langle X\rangle}$ for all the techniques.

The case of the decomposition algorithm is now discussed: for each spectra, three sample function families were used :

- Estimation 1 : the approximation is a linear combination of 21 functions $1 / f^{\gamma i}$ with $\gamma i$ linearly distributed between 1 and 2, 101 Lorentzian functions with $f_{i}$ logarithmically distributed between $1 \mathrm{~Hz}$ and $100 \mathrm{kHz}$ and the uniform function 1 ,

- Estimation 2 : the approximation is a linear combination of the function $1 / f^{2}$, the function $1 / \mathrm{f}, 101$ Lorentzian functions with $f_{i}$ logarithmically distributed between $1 \mathrm{~Hz}$ and $100 \mathrm{kHz}$ and the uniform function 1.

- Estimation 3 : the same as estimation 2 with 130 Lorentzian functions instead of 101

Only the components of the combination with positive non null coefficients have been reported. The main results are that no $1 / \mathrm{f}$ noise exists in the 2 spectra $\mathrm{A}$ and B. For 

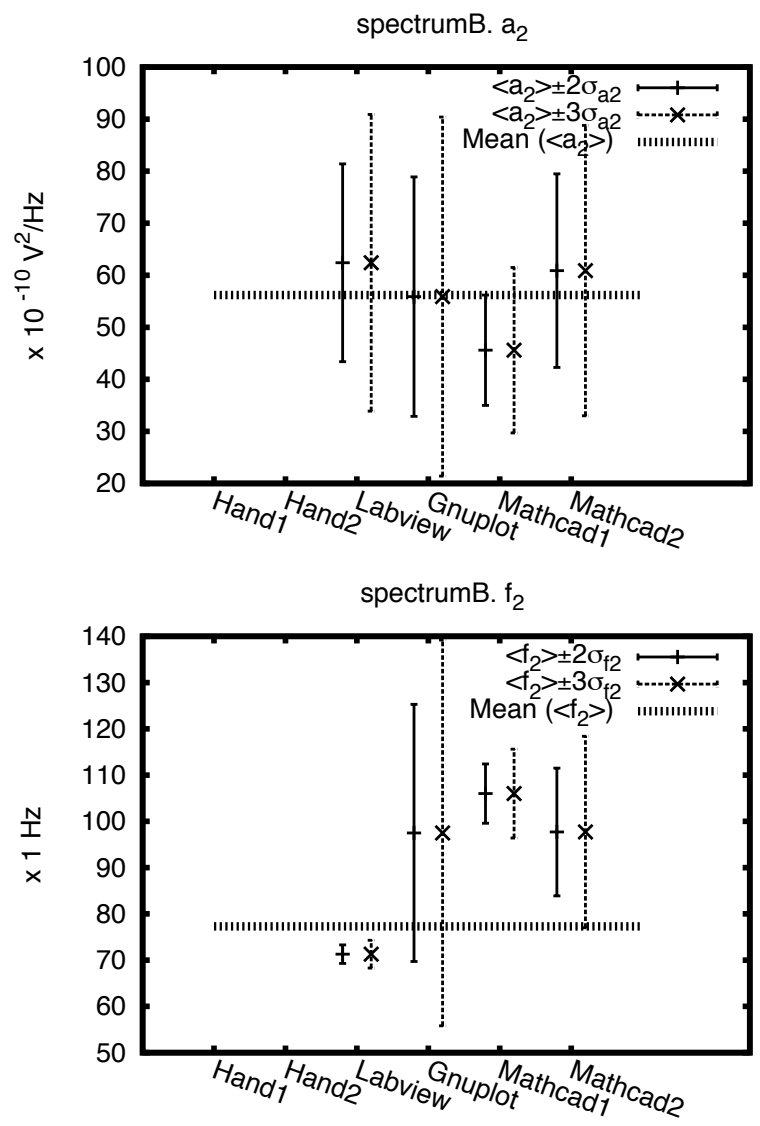

Fig. 5. SpectrumB results for parameters $a_{2}$ and $f_{2} .\langle X\rangle \pm 2 \cdot \sigma_{X}$ $\langle X\rangle \pm 3 \cdot \sigma_{X}$ and $\overline{\langle X\rangle}$ for all the techniques.

spectrumA, only a $1 / f^{1.6}$ was obtained. For spectrumA, 6 lorentzians were reported and 7 lorentzians for spectrumB.

Finally, to compare the different techniques, a relative distance between the measured noise spectral density $S_{V}(f)$ and the approximated functions $S_{V \text { fit }}(f)$ has been calculated according to the following definition :

$$
d=\sum_{f} \frac{\left(S_{V}(f)-S_{V} f i t(f)\right)^{2}}{\left(S_{V}(f)\right)^{2}}
$$

The results are summarized in the following table. As expected, the decomposition technique gives the best results and worst results are obtained by manual estimation. The small differences between software techniques and the decomposition one validate these software techniques.

This study shows that two errors have to be introduced in the uncertainty calculation : the repetability error E1 (mainly due to the operator) estimated from $\sigma_{X}$ and a systematic error E2 (mainly due to the technique, resolution of software slides or design rule) estimated from the standard deviation of all the mean result for one parameter. Finally the expanded uncertainty $u(K)=$

\begin{tabular}{|c|c|c|c|c|c|c|c|}
\hline & $\begin{array}{l}\overrightarrow{\widetilde{Z}} \\
\mathbb{\Xi} \\
\text { ב }\end{array}$ & $\begin{array}{l}\mathcal{T} \\
\underset{\Xi}{\Xi} \\
\text { ב }\end{array}$ & 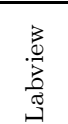 & 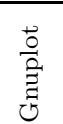 & 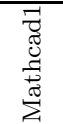 & 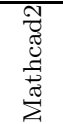 & 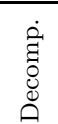 \\
\hline $\mathrm{d} \operatorname{spec} \mathrm{A}$ & 115.4 & 102 & 89.1 & 95.5 & 99.6 & 99.6 & 85.6 \\
\hline d specB & 228.8 & 110.8 & 117.5 & 55.3 & 81.6 & 61 & $\overline{49.2}$ \\
\hline$\frac{\overline{3 \cdot u(K)}}{\langle K\rangle}(\%)$ specA & 12.2 & 12.0 & 14.3 & 16.7 & 13.3 & 13.3 & \\
\hline$\frac{3 \cdot u(K)}{\langle K\rangle}(\%)$ specB & $30.4(*)$ & 14.4 & 18.7 & 18.9 & 17.8 & 22.5 & \\
\hline
\end{tabular}

TABLE I

Comparison of the DifFerent techniques: CAlCulation of THE DISTANCE DEFINED BY EQUATION 3. RELATIVE EXPANDED UNCERTAINTY IN THE ESTIMATION OF THE 1/F NOISE LEVEL FOR SPECTRA A AND B. $(*)$ K VAlue FOR HAND1 WAS NOT TAKEN INTO ACCOUNT IN THE ESTIMATION OF $u(E 2)^{2}$ DUE TO THE OVERESTIMATION.

$k \cdot \sqrt{u(E 1)^{2}+u(E 2)^{2}}$ has been calculated in the table (I) for $k=3$. Between $12 \%$ and $17 \%$ of relative expanded uncertainty is found for spectrum A and between $14 \%$ and $23 \%$ for spectrumB! The larger value obtained for spectrum B may be due to the difficulty to estimate this parameter in the case of lorentzians.

\section{Conclusion}

In this paper, the uncertainty in the estimation of noise parameters has been performed on two kinds of spectra. Bests results has been obtained by a decomposition algorithm. Relative expanded uncertainty between $10 \%$ and $20 \%$ has been obtained for the $1 / \mathrm{f}$ noise level of the 2 spectra.

This work should be completed by choosing several kinds of spectra with only $1 / \mathrm{f}$ noise and white noise with different corner frequencies to estimate the impact of the corner frequency on the expanded uncertainty of $1 / \mathrm{f}$ noise level. The impact of the number of acquisition $N_{\text {avg }}$ could also be study. We propose to make a large inquiry to investigate all these ideas. For that, we encourage you to use the following website to send suggestions, questions, comment and declaration of interest to a new investigation. http://www.greyc.ensicaen.fr/routoure/uncertainty.

\section{REFERENCES}

[1] BIPM : Guide to the expression of uncertainty in measurement http://www.bipm.org/utils/common/documents/jcgm/ JCGM_100_2008_E.pdf

[2] E. Sassier et al., to be published

[3] R. Talmat, H. Achour, B. Cretu, J-M. Routoure, A.Benfdila, R. Carin, N. Collaert, A. Mercha, E. Simoen and C. Claeys, "Assessment of temperature dependence of the low frequency noise in unstrained and strained FinFETs", proceeding of the International conference on Noise and Fluctuations 2011. 